\title{
Radio lobes and X-ray hot spots of the extraordinary microquasar in NGC 7793
}

\author{
Roberto Soria*, Manfred Pakull ${ }^{\dagger}$, Jess Broderick**, Stephane Corbel ${ }^{\ddagger}$ and \\ Christian Motch $^{\dagger}$
}

\author{
${ }^{*}$ MSSL, University College London, Holmbury St Mary, Surrey RH5 6NT, UK \\ ${ }^{\dagger}$ Observatoire Astronomique de Strasbourg, 11 rue de l'Université, 67000 Strasbourg, France \\ ${ }^{* *}$ School of Physics \& Astronomy, University of Southampton, Hampshire SO17 1BJ, UK \\ ¥Université Paris 7 and Service d'Astrophysique, UMR AIM, CEA Saclay, F-91191, Gif sur Yvette,
}

France

\begin{abstract}
We have studied the newly-discovered microquasar in NGC 7793 in radio, optical and $\mathrm{X}$-ray bands. This system comprises a large $(250 \times 120 \mathrm{pc})$ line-emitting optical nebula, detected in $\mathrm{H} \alpha$ and HeII $\lambda$ 4686. The optical nebula coincides with a synchrotron-emitting radio cocoon, with a radio luminosity about 4 times that of Cas A. The central black hole appears as a hard X-ray source with a point-like, blue optical counterpart. Two prominent radio lobes are located at the extremities of the cocoon. Just ahead of the radio lobes, we found two X-ray hot spots, which we interpret as a signature of the bow shock into the interstellar medium. The X-ray hot spots, radio lobes, X-ray core and major axis of the cocoon are well aligned, proving that the system is powered by a jet. From the X-ray data, we estimate a jet power $\sim$ a few $\times 10^{40} \mathrm{erg} \mathrm{s}^{-1}$, active over a timescale $\approx 10^{5}$ yrs. This extraordinary system is a long-sought analog of the Galactic microquasar SS 433.
\end{abstract}

Keywords: X-ray binaries; Jets, outflows, and bipolar flows

PACS: $97.80 . J p$

\section{RADIO GALAXIES AND MICROQUASARS}

The basic physical model for radio lobes in FRII radio galaxies is based on a pair of relativistic, collimated jets emerging from the active black hole $(\mathrm{BH})$. As the jet interacts with and is decelerated by the ambient (interstellar or intergalactic) medium, a reverse shock propagates inwards into the ejected plasma. After crossing the reverse shock, the jet material inflates a cocoon of hot gas, which is less dense but much overpressured with respect to the undisturbed medium. Thus, the cocoon expands supersonically, driving a forward shock (bow shock) into the ambient medium [1, 2, 3, 4].

The cocoon and lobes are the main sources of optically-thin (steep spectrum) synchrotron radio emission, while we expect optically-thick (flat-spectrum) radio emission from the jet near the core. A radio- and sometimes X-ray-luminous hot spot is usually found at the reverse shock, at the end of the jet. This is where most of the bulk kinetic energy of the jet is transferred to a non-thermal population of ultra-relativistic electrons, which cool via synchrotron and synchrotron self-Compton emission. This scenario implies that the peaks of the radio and X-ray emission are spatially coincident. Opticallythin thermal plasma X-ray emission may come instead from the hot, shocked ambient gas in the thin layer between the cocoon and the bow shock, when its expansion is highly supersonic. In this case, the peak of the thermal X-ray emission will appear just in front 

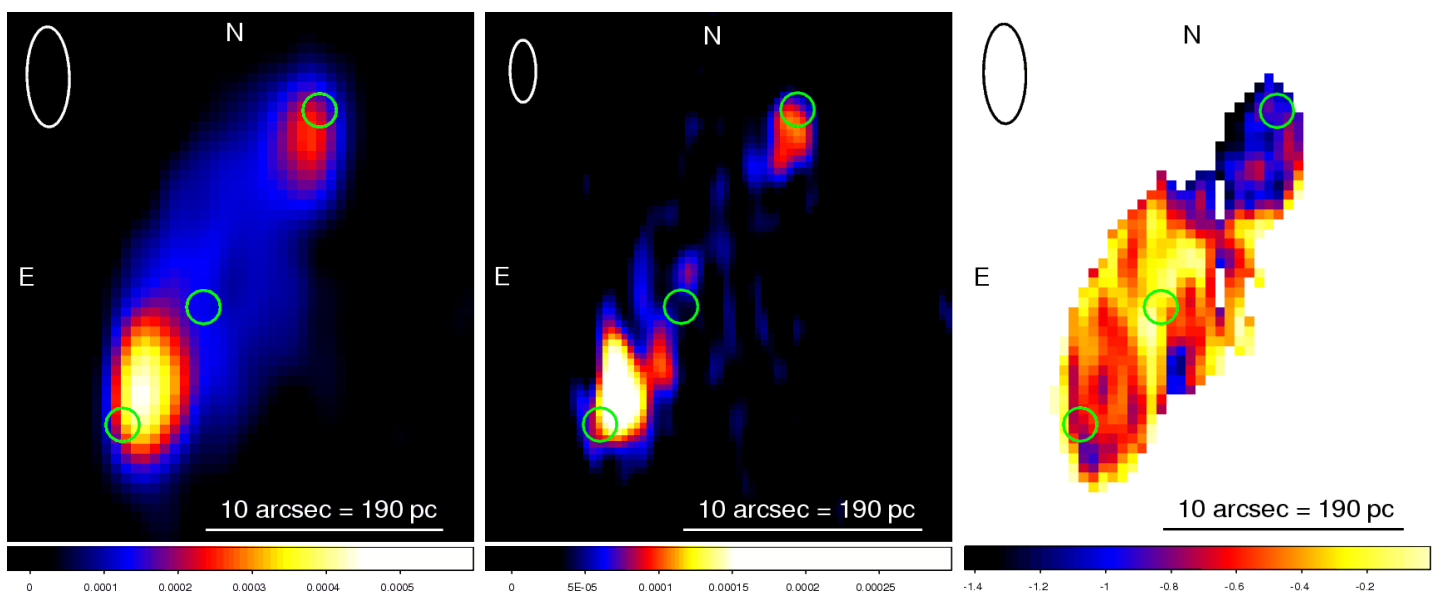

FIGURE 1. Left panel: ATCA map at $5.5 \mathrm{GHz}$, with flux scale in Jy beam ${ }^{-1}$. The overplotted green circles mark the position of the X-ray core and hot spots, from Chandra. Middle panel: ATCA map at $9 \mathrm{GHz}$. Right panel: map of the radio spectral index, inferred from the ratio of the $5.5 \mathrm{GHz}$ and $9 \mathrm{GHz}$ maps, rescaled to the same beam size. Note the two flat-spectrum spurts at the base of the jet.

of the radio lobe, as is the case for example in the nearest radio galaxy, Cen A [5].

There is a scale invariance between the jet emission processes in microquasars (powered by stellar-mass BHs) and in AGN/quasars (powered by supermassive BHs). There is also at least one important difference: microquasars are mostly located in a relatively low-pressure medium as compared to the medium around AGN, when scaling of the jet thrust is taken into account [6]. As a consequence, we expect to see fewer, dimmer cocoons and radio lobes in microquasars than in the most powerful AGN and quasars; but the linear size of those microquasar cocoons and jets can be up to 1000 times larger than in radio galaxies, scaled to their respective $\mathrm{BH}$ masses.

So far, our knowledge of the interaction of microquasar jets with the interstellar medium has largely relied on the Galactic microquasar SS 433 and its surrounding synchrotron-emitting nebula W50 (size $\sim 100 \times 50 \mathrm{pc}$ ). A mildly relativistic $\left(v_{J}=\right.$ $0.27 c$ ), precessing jet acts as a sprinkler that inflates "ear-like" structures, protruding from the more spherical W50 nebula. Most of the jet power $\left(\sim 10^{39}-10^{40} \mathrm{erg} \mathrm{s}^{-1}\right)$ is dissipated in those lobes [7]. Faint evidence of the interaction of relativistic jets with the interstellar medium have been found in a few other, less powerful Galactic microquasars (e.g., Cyg X-1: [8]; Cyg X-3: [9]; GRS 1915+105: [10]). On a larger scale, huge (size $\gtrsim 100 \mathrm{pc}$ ) ionized nebulae have been found around a significant fraction of ultraluminous $\mathrm{X}$-ray sources in nearby galaxies [11, 12, 13]. Such nebulae emit optical lines typical of shock-ionized gas, and in a few cases, synchrotron radio emission [14, 15]. The derived ages ( $\gtrsim$ a few $\left.10^{5} \mathrm{yrs}\right)$ and energy content $\left(\sim 10^{52}-10^{53} \mathrm{erg}\right)$ are too large for ordinary supernova remnants, and suggest jet/wind inflation with a mechanical power $\sim 10^{39}$ $10^{40} \mathrm{erg} \mathrm{s}^{-1}$, comparable with the X-ray luminosities [11]. However, no direct X-ray or radio evidence of a collimated jet had been found, until recently. 

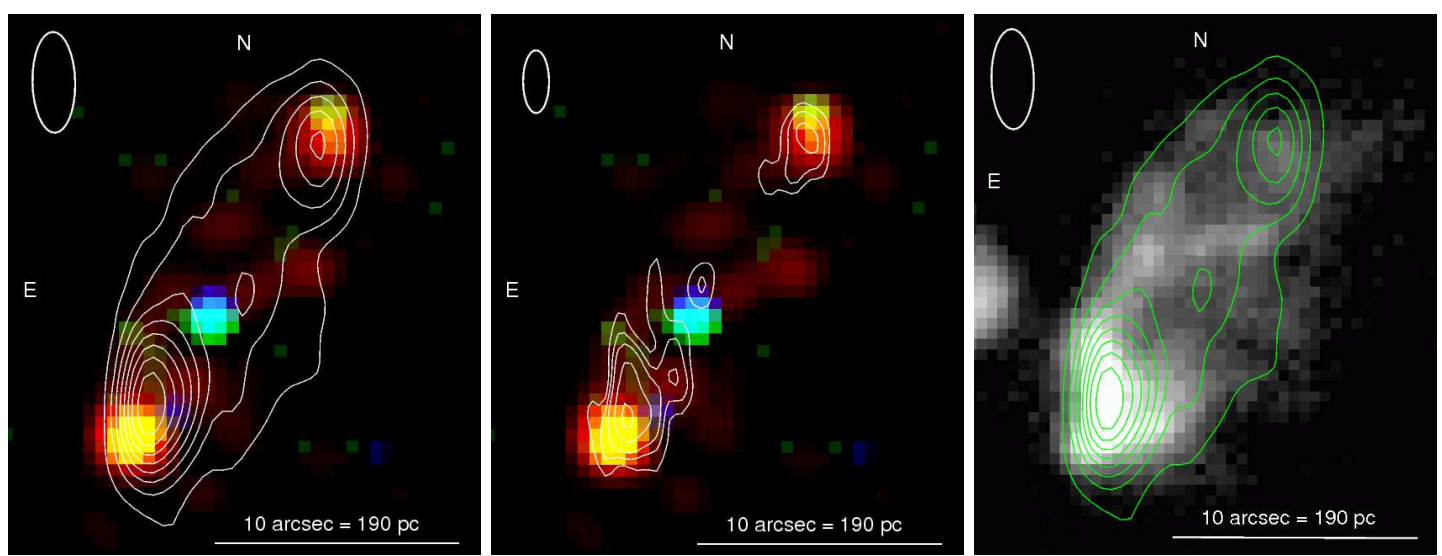

FIGURE 2. Left panel: Chandra/ACIS X-ray colour map, with ATCA 5.5-GHz radio flux contours superimposed. Red $=0.3-1 \mathrm{keV}$; green $=1-2 \mathrm{keV}$; blue $=2-8 \mathrm{keV}$. Contour fluxes are at $5 \times 10^{-5}, 10^{-4}$, $1.5 \times 10^{-4}, 2 \times 10^{-4}, 2.5 \times 10^{-4}, 3 \times 10^{-4}, 3.5 \times 10^{-4}, 4 \times 10^{-4} \mathrm{Jy} \mathrm{beam}^{-1}$. Middle panel: Chandra/ACIS X-ray colour map, with ATCA 9-GHz radio flux contours superimposed. Contour fluxes are at $5 \times 10^{-5}$, $7.5 \times 10^{-5}, 10^{-4}, 1.5 \times 10^{-4}, 2 \times 10^{-4} \mathrm{Jy} \mathrm{beam}^{-1}$. Right panel: continuum-subtracted $\mathrm{H} \alpha$ image from the $1.5 \mathrm{~m}$ CTIO telescope; ATCA 5.5-GHz radio flux contours superimposed.

\section{THE NEWLY-DISCOVERED SS433-LIKE MICROQUASAR}

An extraordinary radio/optical/X-ray microquasar was recently discovered [12] in the Sculptor galaxy NGC $7793(d \approx 3.9 \mathrm{Mpc})$. It has: a large $(\approx 250 \times 120 \mathrm{pc})$ shock-ionized nebula, emitting in the radio bands, $\mathrm{H} \alpha$ and HeII $\lambda 4686$; prominent FRII-like radio lobes, more luminous than the rest of the cocoon; an X-ray core, presumably the location of the active $\mathrm{BH}$; a blue optical counterpart to the X-ray core; a pair of X-ray hot spots, aligned with the radio lobes and the X-ray core, typical signature of energy transport via a collimated jet interacting with the interstellar medium. The system resembles the famous Galactic microquasar SS 433, but on an even grander scale. It was previously classified as the unusual supernova remnant S26 in [16] and [17].

Radio properties. We observed the source simultaneously in the $3 \mathrm{~cm}$ and $6 \mathrm{~cm}$ bands with the Australia Telescope Compact Array, on 2009 Aug 6-7, for about 18 hrs. Details of the observations and data analysis will be presented in a forthcoming paper (Soria et al. 2010, in prep.). We extracted radio maps at the effective frequencies of 5480 $\mathrm{MHz}$ and $8990 \mathrm{MHz}$ (Fig. 1); the beam sizes are $4.16^{\prime \prime} \times 1.76^{\prime \prime}$, and $2.58^{\prime \prime} \times 1.07^{\prime \prime}$ respectively (Briggs robustness parameter of 0.5). Two bright lobes clearly stand out at the extremities of the large cocoon, at both frequencies. At $5.5 \mathrm{GHz}$, the peak flux in the Southern lobe is $\approx 0.45 \mathrm{mJy} \mathrm{beam}^{-1}$; in the Northern lobe, $\approx 0.26 \mathrm{mJy} \mathrm{beam}^{-1}$. The total flux at $5.5 \mathrm{GHz}$ is $\approx 2 \mathrm{mJy}$, corresponding to almost 4 times the luminosity of Cas A. At $9 \mathrm{GHz}$, the peak flux in the Southern lobe is $\approx 0.21 \mathrm{mJy}^{\text {beam }}{ }^{-1}$; in the Northern lobe, $\approx 0.11 \mathrm{mJy}$ beam $^{-1}$. The spectral index (Fig. 1 , right) is $\approx 0$ at the base of the jets, on either side of the X-ray core; it steepens to $\approx-0.7$ in the Southern lobe and $\approx-1$ in the Northern lobe. The projected distance between the peak emission in the two lobes (that is, between the reverse shocks at the jet extremities) is $\approx 13.5^{\prime \prime} \approx 250 \mathrm{pc}$. 
TABLE 1. Best-fitting spectral parameters for the (combined) hot spot $\mathrm{X}$-ray emission. The XSPEC model is $w_{a b} s_{\mathrm{Gal}}{ }^{*} w a b s *($ ray + ray $)$. Errors are $90 \%$ confidence level for 1 interesting parameter $\left(\Delta \chi^{2}=2.7\right)$.

\begin{tabular}{lr}
\hline Parameter & Value \\
\hline$N_{H, \text { Gal }}$ & $1.2 \times 10^{20} \mathrm{~cm}^{-2}(\mathrm{fixed})$ \\
$N_{H}$ & $<2.9 \times 10^{20} \mathrm{~cm}^{-2}$ \\
$k T_{1}$ & $0.26_{-0.08}^{+0.06} \mathrm{keV}$ \\
$N_{1}$ & $\left(2.4_{-0.7}^{+0.8}\right) \times 10^{-6}$ \\
$k T_{2}$ & $0.96_{-0.20}^{+0.32} \mathrm{keV}$ \\
$N_{2}$ & $\left(2.0_{-0.7}^{+0.8}\right) \times 10^{-6}$ \\
\hline$f_{0.3-8}$ & $8.8 \times 10^{-15} \mathrm{erg} \mathrm{cm}^{-2} \mathrm{~s}^{-1}$ \\
$L_{0.3-8}$ & $1.7 \times 10^{37} \mathrm{erg} \mathrm{s}^{-1}$ \\
\hline C-statistic & 10.37 using 18 PHA bins and 14 degrees of freedom.
\end{tabular}

X-ray properties. From a 49-ks Chandra archival observation taken on 2003 Sep 7, we found an aligned triplet of point-like sources [12]. The X-ray core (Fig. 2,3) has a hard spectrum (photon index $\Gamma \approx 1.7$ ), with an emitted luminosity $L_{0.3-8} \approx 6 \times 10^{36}$ erg s$~^{-1}$, consistent with a BH in the low/hard state (Pakull et al. 2010, in prep.). The two hot spots have a much softer spectrum (Fig. 2,3), and are well fitted (Cash statistics $=10.4$ over 14 dof) by a 2-component thermal plasma model with $k T_{1} \approx 0.25 \mathrm{keV}$ and $k T_{2} \approx 0.95 \mathrm{keV}$, and negligible intrinsic absorption (Table 1). The emitted X-ray luminosities are $L_{0.3-8} \approx 5 \times 10^{36} \mathrm{erg} \mathrm{s}^{-1}$ and $L_{0.3-8} \approx 11 \times 10^{36} \mathrm{erg} \mathrm{s}^{-1}$ for the Northern and Southern hot spot, respectively (in agreement with the ratio of radio luminosities). Simple or broken power-law models do not give acceptable fits (Cash statistics $=25.5$ over 15 dof); moreover, they would require an unphysically steep slope $(\Gamma \approx 6)$ combined with high intrinsic column densities $\left(N_{H} \approx 5 \times 10^{21} \mathrm{~cm}^{-2}\right)$. We conclude that the hot spot spectra are not dominated by synchrotron or synchrotron self-Compton emission. Moreover, the projected distance between the X-ray hot spots is $\approx 15^{\prime \prime} \approx 290 \mathrm{pc}$, slightly larger than the distance between the radio lobes. We interpret the X-ray hot spots as optically-thin thermal plasma emission from the shocked ISM gas between the radio lobes and the bow shock. From the maximum size of the unresolved hot spots, we infer that the density of the X-ray-emitting gas is $\gtrsim 1 \mathrm{~cm}^{-3}$. A shocked-gas temperature $\approx 1 \mathrm{keV}$ corresponds to a bow shock velocity $v_{b s} \approx 900 \mathrm{~km} \mathrm{~s}^{-1}$. For an ISM of uniform density $\rho$, the distance from the core to the bow shock $l_{b s} \sim \rho^{-1 / 5} Q^{1 / 5} t^{3 / 5}$ and its velocity $v_{b s} \approx(3 / 5)\left(l_{b s} / t\right)$, where $Q$ is the jet power and $t$ the age of the source [4]. Neglecting projection effects, this suggests a characteristic age $\approx 10^{5} \mathrm{yrs}$ and a jet power $\sim$ a few $\times 10^{40} \mathrm{erg} \mathrm{s}^{-1}$. The stronger X-ray and radio emission from the Southern hot spot and lobe, and their shorter distance to the core, are consistent with a higher density of the ISM on that side of the core. There is also faint X-ray emission projected over the whole surface of the cocoon, with even softer colours than in the lobes (although the number of detected counts is too low for spectral fitting); this diffuse emission is consistent with shock velocities $\approx 300 \mathrm{~km} \mathrm{~s}^{-1}$. 


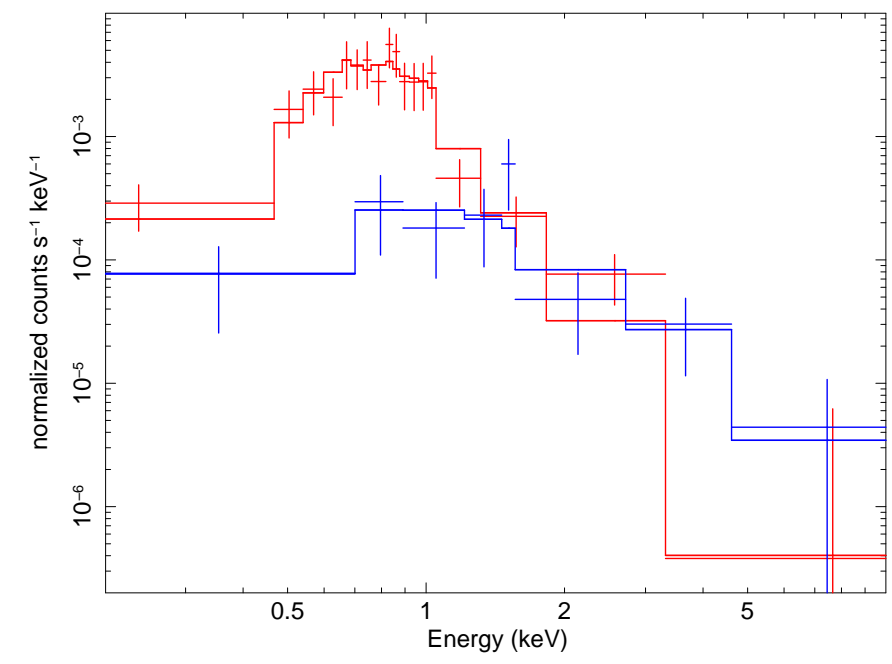

FIGURE 3. Red datapoints and best-fitting model: combined X-ray spectrum of the hot spots, fitted with a two-temperature thermal plasma model (best-fitting parameters listed in Table 1). Blue datapoints and best-fitting model: X-ray spectrum of the core, fitted with a power-law of photon index $\Gamma=1.7$.

Optical properties. Optical images from the $1.5 \mathrm{~m}$ CTIO telescope show an $\mathrm{H} \alpha$ emission nebula with similar size to the radio nebula (Fig. 2, right panel). From integral field spectra taken at the $2.3 \mathrm{~m}$ ANU telescope, we find that the $\mathrm{H} \alpha$ line is broad (full width at zero intensity $\approx 250 \mathrm{~km} \mathrm{~s}^{-1}$ ). Moreover, using VLT data, we have recently found (Pakull et al. 2010, in prep.) diffuse emission in the HeII $\lambda 4686$ recombination line, with a flux $\approx 10 \%$ of the $\mathrm{H} \beta$ flux. It is the largest known HeIII region in the local universe. Considering the relative weakness of the central X-ray source, the most likely explanation is shock ionization with a large shock velocity $\left(\approx 300 \mathrm{~km} \mathrm{~s}^{-1}\right)$. Using radiative shock model computations, we estimate a mechanical luminosity $\approx 6 \times 10^{40}$ $\mathrm{erg} \mathrm{s}^{-1}$. The X-ray core has a point-like blue counterpart, with $B \sim 23 \operatorname{mag}\left(M_{B} \sim-5\right.$ mag), consistent with an early-type donor star or a bright accretion disk. The optical line emission is also stronger on the Southern side of the nebula, in agreement with the radio and X-ray morphology. The shock velocity inferred from the optical spectra is much lower than that inferred from the X-ray temperatures. By analogy with wellstudied supernova remnants, for example N49 in the LMC [18, 19], we suggest that we have a clumpy medium, with the X-ray emission coming from higher-velocity shocks in lower-density material, and the optical lines coming from slower shocks in higherdensity embedded clouds.

\section{ACKNOWLEDGMENTS}

We thank Tasso Tzioumis for his assistance when we prepared the ATCA observations, and Mike Dopita for comments and for his taking of an optical spectrum for us, from the ANU 2.3m telescope. RS acknowledges hospitality at Tsing Hua University (Taiwan), at Mount Stromlo Observatory, and at the University of Sydney, during the completion of this work. 


\section{REFERENCES}

1. P. A. G. Scheuer, MNRAS 166, 513-528 (1974).

2. R. D. Blandford, and M. J. Rees, MNRAS 169, 395-415 (1974).

3. S. Rawlings, and R. Saunders, Nature 349, 138-140 (1991).

4. C. R. Kaiser, and P. Alexander, MNRAS 305, 707-723 (1999).

5. R. P. Kraft, P. E. J. Nulsen, M. Birkinshaw, et al., ApJ 665, 1129-1137 (2007).

6. S. Heinz, A\&A 338, L40-L43 (2002)

7. M. C. Begelman, S. P. Hatchett, C. F. McKee, C. L. Sarazin, and J. Arons, ApJ 238, 722-730 (1980).

8. E. Gallo, R. P. Fender, C. R. Kaiser, et al., Nature 436, 819-821 (2005).

9. J. Martí, et al., $A \& A$ 439, 279-285 (2005).

10. C. R. Kaiser, K. F. Gunn, C. Brocksopp, and J. L. Sokoloski, ApJ 612, 332-341 (2004).

11. M. W. Pakull, F. Grisé, and C. Motch, 2006, in Populations of High Energy Sources in Galaxies, proc. of the IAU Symposium 230, Cambridge University Press, Cambridge, 2006, pp. 293-297.

12. M. W. Pakull, and F. Grisé, in A Population Explosion: the Nature \& Evolution of X-ray Binaries in Diverse Environments, AIP Conf. Proc. 1010, AIP, New York, 2008, pp. 303-307.

13. F. Grisé, M. W. Pakull, R. Soria, et al., A\&A 486, 151-163 (2008).

14. N. A. Miller, R. F. Mushotzky, and S. G. Neff, ApJ 623, L109-L112 (2005).

15. R. Soria, et al., MNRAS 368, 1527-1539 (2006).

16. W. P. Blair, and K. S. Long, ApJS 108, 261-277 (1997).

17. T. G. Pannuti, N. Duric, C. K. Lacey, et al., ApJ 565, 966-981 (2002).

18. O. Vancura, W. P. Blair, K. S. Long, and J. C. Raymond, ApJ 394, 158-173 (1992).

19. S. Park, D. N. Burrows, and G. P. Garmire, ApJ 586, 210-223 (2003). 